\title{
Erratum to: Interpreting findings from Mendelian randomization using the MR-Egger method
}

\author{
Stephen Burgess $^{1,2}$ (1) $\cdot$ Simon G. Thompson ${ }^{2}$
}

Published online: 29 June 2017

(C) Springer Science+Business Media B.V. 2017

\section{Erratum to: Eur J Epidemiol}

\section{DOI 10.1007/s10654-017-0255-x}

Figure 6 as originally published was incorrect-arrows were orientated from the risk factor to the confounder, whereas they should have been orientated from the con- founder to the risk factor. This has now been corrected. The authors regret the error (Fig. 6).

The online version of the original article can be found under doi:10.1007/s10654-017-0255-x.

Stephen Burgess

sb452@medschl.cam.ac.uk

1 MRC Biostatistics Unit, Cambridge Institute of Public Health, University of Cambridge, Forvie Site, Robinson Way, Cambridge CB2 OSR, UK

2 Department of Public Health and Primary Care, University of Cambridge, Cambridge, UK 

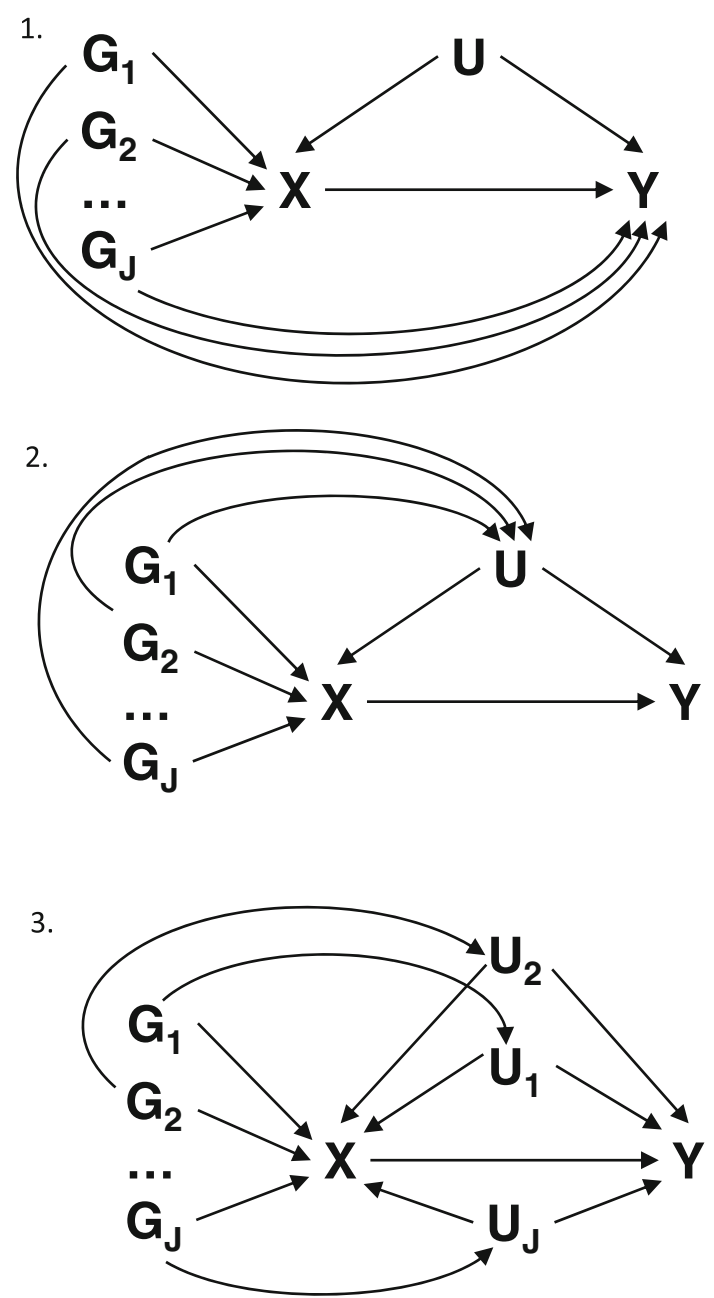

Fig. 6 Potential violations of the InSIDE assumption. Top panel pleiotropic effects act directly on the outcome (InSIDE satisfied); middle panel pleiotropic effects act on the outcome via single confounder (InSIDE violated); bottom panel pleiotropic effects act on the outcome via different confounders (InSIDE still violated). Arrows from the genetic variants to the risk factor may not be present for all variants; some variants may affect the confounder directly and not the risk factor. Notation: $G_{1}, G_{2}, \ldots, G_{J}$, genetic variants; $X$, risk factor; $Y$, outcome; $U$, confounder. Pleiotropic effects are signified by curved arrows 